\title{
Front Matter: Volume 9648
}

, "Front Matter: Volume 9648," Proc. SPIE 9648, Electro-Optical and Infrared Systems: Technology and Applications XII; and Quantum Information Science and Technology, 964801 (28 October 2015); doi: 10.1117/12.2217600

SPIE. Event: SPIE Security + Defence, 2015, Toulouse, France 


\title{
Electro-Optical and Infrared Systems: Technology and Applications XII; and Quantum Information Science and Technology
}

\author{
David A. Huckridge \\ Reinhard Ebert \\ Mark T. Gruneisen \\ Miloslav Dusek \\ John G. Rarity \\ Editors
}

22-23 September 2015

Toulouse, France

Sponsored by

SPIE

Cooperating Organisations

European Optical Society

Optitec (France)

Route des Lasers (France)

Published by

SPIE 
The papers in this volume were part of the technical conference cited on the cover and title page. Papers were selected and subject to review by the editors and conference program committee. Some conference presentations may not be available for publication. Additional papers and presentation recordings may be available online in the SPIE Digital Library at SPIEDigitallibrary.org.

The papers reflect the work and thoughts of the authors and are published herein as submitted. The publisher is not responsible for the validity of the information or for any outcomes resulting from reliance thereon.

Please use the following format to cite material from these proceedings:

Author(s), "Title of Paper," in Electro-Optical and Infrared Systems: Technology and Applications XII; and Quantum Information Science and Technology, edited by David A. Huckridge, Mark T. Gruneisen, Miloslav Dusek, Reinhard Ebert, John G. Rarity, Proceedings of SPIE Vol. 9648 (SPIE, Bellingham, WA, 2015) Six-digit Article CID Number.

ISSN: 0277-786X

ISSN: 1996-756X (electronic)

ISBN: 9781628418583

Published by

SPIE

P.O. Box 10, Bellingham, Washington 98227-0010 USA

Telephone +1 3606763290 (Pacific Time) · Fax +1 3606471445

SPIE.org

Copyright (C) 2015, Society of Photo-Optical Instrumentation Engineers.

Copying of material in this book for internal or personal use, or for the internal or personal use of specific clients, beyond the fair use provisions granted by the U.S. Copyright Law is authorized by SPIE subject to payment of copying fees. The Transactional Reporting Service base fee for this volume is $\$ 18.00$ per article (or portion thereof), which should be paid directly to the Copyright Clearance Center (CCC), 222 Rosewood Drive, Danvers, MA 01923. Payment may also be made electronically through CCC Online at copyright.com. Other copying for republication, resale, advertising or promotion, or any form of systematic or multiple reproduction of any material in this book is prohibited except with permission in writing from the publisher. The CCC fee code is $0277-786 \mathrm{X} / 15 / \$ 18.00$.

Printed in the United States of America.

Publication of record for individual papers is online in the SPIE Digital Library.

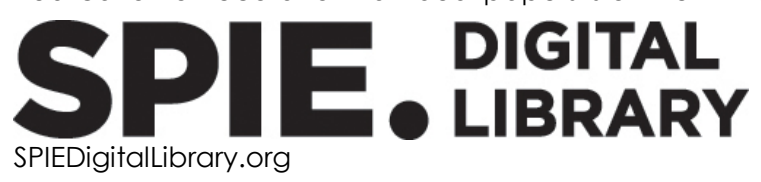

Paper Numbering: Proceedings of SPIE follow an e-First publication model, with papers published first online and then in print. Papers are published as they are submitted and meet publication criteria. A unique citation identifier (CID) number is assigned to each article at the time of the first publication. Utilization of CIDs allows articles to be fully citable as soon as they are published online, and connects the same identifier to all online, print, and electronic versions of the publication. SPIE uses a six-digit CID article numbering system in which:

- The first four digits correspond to the SPIE volume number.

- The last two digits indicate publication order within the volume using a Base 36 numbering system employing both numerals and letters. These two-number sets start with 00, 01, 02, 03, 04, $05,06,07,08,09,0 A, 0 B \ldots$. 0Z, followed by 10-1Z, 20-2Z, etc.

The CID Number appears on each page of the manuscript. The complete citation is used on the first page, and an abbreviated version on subsequent pages. 


\title{
Contents
}

\author{
vii Authors \\ ix Conference Committee
}

\section{Part A Electro-Optical and Infrared Systems: Technology and Applications}

\section{ACTIVE AND SPECTRAL SYSTEMS}

964802 Simulation of a multispectral, multicamera, off-road autonomous vehicle perception system with Virtual Autonomous Navigation Environment (VANE) (Invited Paper) [9648-1]

964803 Time-resolved multispectral imaging of combustion reactions [9648-2]

964804 Stand-off detection of liquid thin films using active mid-infrared hyperspectral imaging (Best Student Paper) [9648-3]

964805 Performance assessment of simulated 3D laser images using Geiger-mode avalanche photo-diode: tests on simple synthetic scenarios [9648-4]

964806 Long-range concealed object detection through active covert illumination [9648-5]

\section{BROADBAND EO SYSTEMS}

964807 The use of algorithmic behavioural transfer functions in parametric EO system performance models (Invited Paper) [9648-6]

964809 Compressive sensing applications for single detector rosette scanning infrared seekers [9648-8]

9648 OA A probabilistic blur detection approach for the autofocus of infrared images [9648-9]

\section{OPTICAL TECHNOLOGIES}

9648 OD Thickness and air gap measurement of assembled IR objectives [9648-12]

9648 OE Digital holographic interferometer with correction of distortions [9648-13]

\section{ELECTRO-OPTICAL DETECTORS}

9648 OG A true differential pyroelectric IR detector with improved D* [9648-15]

$9648 \mathrm{OH}$ A new generation of small pixel pitch/SWaP cooled infrared detectors [9648-16] 
9648 ol Vehicle tracking in wide area motion imagery from an airborne platform (Invited Paper) [9648-17]

$96480 \mathrm{~J}$ Information hiding techniques for infrared images: exploring the state of the art and challenges [9648-18]

9648 OK A multiscale contrast direction adaptation approach for the fusion of multispectral and multifocus infrared images [9648-19]

$9648 \mathrm{OL}$ Graph Laplacian regularization based edge-preserving background estimation for single frame small target detection [9648-21]

POSTER SESSION

9648 OM Test and analysis of spectral response for UV image intensifier [9648-25]

$9648 \mathrm{OP}$ Some design considerations for high-performance infrared imaging seeker [9648-29]

$96480 Q$ Design of a long service life integration CES [9648-30]

9648 OR Fast measurement of temporal noise of digital camera's photosensors [9648-31]

9648 OS Modeling of digital information optical encryption system with spatially incoherent illumination [9648-32]

\section{Part B Quantum Information Science and Technology}

\section{SECURITY OF QUANTUM INFORMATION SYSTEMS}

9648 OU Practical aspects of security certification for commercial quantum technologies [9648-42]

9648 OV Quantum hacking on a practical continuous-variable quantum cryptosystem by inserting an external light [9648-43]

\section{QUANTUM CRYPTOGRAPHY: PROTOCOLS AND IMPLEMENTATIONS}

$96480 \mathrm{X}$ Bridging the gap between theory and practice in quantum cryptography (Invited Paper) [9648-45]

9648 OY Establishing security of quantum key distribution without monitoring disturbance (Invited Paper) [9648-46]

$96480 Z$ Quantum cryptography with an ideal local relay (Invited Paper) [9648-47] 
$964811 \quad$ Deploying quantum light sources on nanosatellites II: lessons and perspectives on CubeSat spacecraft (Invited Paper) [9648-49]

964813 A low bias variation SPAD-based pixel for a quantum random generator [9648-51]

964814 Heralded single-photon source from spontaneous four-wave mixing process in lossy waveguides [9648-52]

\section{QUANTUM COMPUTATION AND QUANTUM INFORMATION THEORY}

964815 Benchmarking D-Wave quantum annealing systems: some challenges (Invited Paper) [9648-53]

964816 Performance of quantum annealing hardware (Invited Paper) [9648-54]

964818 A complete classification of quantum public-key encryption protocols [9648-57] 
Proc. of SPIE Vol. $9648964801-6$

Downloaded From: https://www.spiedigitallibrary.org/conference-proceedings-of-spie on 25 Apr 2023 Terms of Use: https://www.spiedigitallibrary.org/terms-of-use 


\title{
Authors
}

Numbers in the index correspond to the last two digits of the six-digit citation identifier (CID) article numbering system used in Proceedings of SPIE. The first four digits reflect the volume number. Base 36 numbering is employed for the last two digits and indicates the order of articles within the volume. Numbers start with 00, 01, 02, 03, 04, 05, 06, 07, 08, 09, 0A, 0B...0Z, followed by 10-1Z, 20-2Z, etc.

\author{
Alleaume, Romain, OV \\ Andersen, Ulrik L., $\mathrm{OZ}$ \\ Aytaç, Tayfun, 09, 0K \\ Baan, Jan, 01 \\ Bai, Kun, OL \\ Bedington, R., 11 \\ Bondareva, Alyona P., OS \\ Botta, Marco, OJ \\ Braunstein, Samuel L., $0 Z$ \\ Briottet, Xavier, 05 \\ Broadbent, Laurence, 06 \\ Brunner, A., $\mathrm{OH}$ \\ Cakir, Serdar, 09, OA, OK \\ Caselunghe, Dario, OU \\ Castelein, P., $\mathrm{OH}$ \\ Cavagnino, Davide, OJ \\ Cetin, A. Enis, OA \\ Chamberland, Martin, 03 \\ Chambers, David R., 02 \\ Cheng, C., 11 \\ Cheremkhin, Pavel A., OR, OS \\ Christie, A., 04 \\ Coyac, Antoine, 05 \\ Curty, Marcos, OX \\ Dariel, A., $\mathrm{OH}$ \\ Deng, Loulou, $0 Q$ \\ Doctor, Alan, OG \\ Domergue, Mathias, OU \\ Durak, K., 11 \\ Durst, Phillip J., 02 \\ Eendebak, Pieter T., 01 \\ Espuno, L., $\mathrm{OH}$ \\ Evtikhiev, Nikolay N., OR \\ Fan, Jinxiang, OP \\ Farley, Vincent, 03 \\ Feng, Cheng, OM \\ Gagnon, Marc-André, 03 \\ Gasparini, Leonardo, 13 \\ Gassaway, Jason, 02 \\ Gehring, Tobias, $\mathrm{OZ}$ \\ Goodin, Christopher, 02 \\ Gravrand, O., $\mathrm{OH}$ \\ Grieve, J., 11 \\ Guyot, Éric, 03 \\ Hagerman, Michael, OU \\ Hales, Ian J., 06 \\ Hansen, Mark F., 06 \\ Hart, Randall, OU \\ Hayford, Don, OU
}

Heim, Bettina, 16

Hespel, Laurent, 05

Hickman, Duncan L., 07

Houlmann, Raphaël, OU

Howle, C. R., 04

$\mathrm{Hu}$, Xinchao, $O Q$

Huang, Jianxiong, $O P$

Huot, Alexandrine, 03

Jacobsen, Christian S., OZ

Jahjah, Karl-Alexandre, 03

Karall, Onur A., OK

Kerlain, A., $\mathrm{OH}$

Kessler, A., $\mathrm{OH}$

Koashi, Masato, OY

Krasnov, Vitaly V., OR, OS

Kumar, Rupesh, OV

Lagueux, Philippe, 03

Langehanenberg, P., OD

Larsen, J., 11

Lee, S. T., 04

Legré, Matthieu, OU

Lim, Charles Ci Wen, OX

Ling, A., 11

Liu, Jian, OM

Liv, Mingna, OL

Lo, Hoi-Kwong, OX

Lueerss, B., OD

LV, Yang, OM

Maidment, L., 04

Marcotte, Frédérick, 03

Massari, Nicola, 13

McCandlish, Todd, OU

McGeoch, Catherine C., 15

Mei, Zhiwu, OQ

Meneghetti, Alessio, 13

Mizutani, Akihiro, OX

Nejati, Hossein, 0J

Oi, D., 11

Ottaviani, Carlo, $\mathrm{OZ}$

Pacaud, $\mathrm{O}$., $\mathrm{OH}$

Page, Jean-Benoît, OU

Péré-Laperne, N., $\mathrm{OH}$

Perenzoni, Daniele, 13

Pinto, Armando N., 14

Pirandola, Stefano, $\mathrm{OZ}$

Pomponiu, Victor, OJ

Pulkin, S. A., OE

Qi, Bing, OX

Qian, Yunsheng, OM 
Qin, Hao, OV

Reibel, Y., $\mathrm{OH}$

Reid, D. T., 04

Riviere, Nicolas, 05

Rodin, Vladislav G., OR, OS

Rønnow, Troels F., 16

Roumegoux, J., $\mathrm{OH}$

Rubaldo, L., $\mathrm{OH}$

Savary, Simon, 03

Sevryugin, A. A., OE

Silva, Nuno A., 14

Smith, Melvyn, 06

Smith, Moira I., 07

Song, Qiong, OL

Soucarros, Mathilde, oU

Spedalieri, Gaetana, $\mathrm{OZ}$

Starikov, Rostislav S., OR, OS

Starikov, Sergey N., OR, OS

Steiger, Damian S., 16

Stoppa, David, 13

Stucki, Damien, OU

Tamaki, Kiyoshi, OX

Tan, Y. C., 11

Tomasi, Alessandro, 13

Tourville, Maurice, oU

Tremblay, Pierre, 03

Troyer, Matthias, 16

Truong-Cao, E., 11

Tu, Zhijun, OQ

Tursunov, I. M., OE

Uzeler, Hande, 09

van Eekeren, Adam W. M., Ol

van Huis, Jasper R., Ol

Venediktov, D. V., OE

Venediktov, V. YU., OE

Walenta, Nino, oU

Wang, Yuehuang, $\mathrm{OL}$

Williamson, David R, 06

Wolterman, Richard, OU

Wu, Chenmiao, 18

WU, Jiandong, $\mathrm{OL}$

$\mathrm{Xu}$, Feihu, OX

Yang, Li, 18

Zhang, Yijun, OM

Zhang, Z., 04 


\title{
Conference Committee
}

\author{
Symposium Chairs
}

David H. Titterton, UK Defense Academy (United Kingdom)

Symposium Co-chair

Reinhard Ebert, Fraunhofer-Institut für Optronik, Systemtechnik und Bildauswertung (Germany)

\section{Part A: Electro-Optical and Infrared Systems: Technology and Applications}

Conference Chairs

David A. Huckridge, Ridgeway Consulting (United Kingdom)

Reinhard Ebert, Fraunhofer-Institut für Optronik, Systemtechnik und Bildauswertung (Germany)

Conference Programme Committee

Christopher C. Alexay, StingRay Optics, LLC (United States) Jan Y. Andersson, Acreo Swedish ICT AB (Sweden) Gisele Bennett, Georgia Institute of Technology (United States) Piet Bijl, TNO Defence, Security and Safety (Netherlands) Rainer Breiter, AIM INFRAROT-MODULE GmbH (Germany) Gordon A. Cain, Vision4ce Ltd. (United Kingdom) David J. Clarke, Placing Value Co., Ltd (Thailand) Gérard Destéfanis, Commissariat à l'Énergie Atomique (France) Judith Dijk, TNO Defence, Security and Safety (Netherlands) Jean-Claude L. Fontanella, Thales Optronique S.A.S. (France) Natan S. Kopeika, Ben-Gurion University of the Negev (Israel) Robert A. Lamb, SELEX ES (United Kingdom) Stephen T. Lee, Thales Optronics Ltd. (United Kingdom) José Manuel López-Alonso, Universidad Complutense de Madrid (Spain)

John F. Parsons, Thales UK Ltd. (United Kingdom)

Stanley R. Rotman, Ben-Gurion University of the Negev (Israel)

Armin L. Schneider, Institut Franco-Allemand de Recherches de SaintLouis (France)

Philip J. Soan, Defence Science and Technology Laboratory (United Kingdom) 
Session Chairs

Active and Spectral Systems

Stephen T. Lee, Thales Optronics Ltd. (United Kingdom)

Broadband EO Systems

Jasper R. van Huis, TNO (Netherlands)

Optical Technologies

David A. Huckridge, Ridgeway Consulting (United Kingdom)

Electro-Optical Detectors

Reinhard Ebert, Fraunhofer-Institut für Optronik, Systemtechnik und Bildauswertung (Germany)

Processing

Helge Buersing, Fraunhofer-Institut für Optronik, Systemtechnik und Bildauswertung (Germany)

\section{Part B: Quantum Information Science and Technology}

\section{Conference Chairs}

Mark T. Gruneisen, Air Force Research Laboratory (United States) Miloslav Dusek, Palacký University Olomouc (Czech Republic) John G. Rarity, University of Bristol (United Kingdom)

Conference Programme Committee

Jan Bouda, Masaryk University (Czech Republic)

Robert W. Boyd, University of Ottawa (Canada)

Gerald S. Buller, Heriot-Watt University (United Kingdom)

Ryan M. Camacho, Sandia National Laboratories (United States)

John D. Gonglewski, European Office of Aerospace Research and Development (United Kingdom)

Gregory S. Kanter, NuCrypt LLC (United States)

Prem Kumar, Northwestern University (United States)

Norbert Lütkenhaus, University of Waterloo (Canada)

Vadim V. Makarov, University of Waterloo (Canada)

Ronald E. Meyers, U.S. Army Research Laboratory (United States)

Momtchil Peev, Austrian Research Centers GmbH - ARC (Austria)

Renato Renner, ETH Zürich (Switzerland)

Andrew J. Shields, Toshiba Research Europe Ltd. (United Kingdom) Rupert Ursin, Austrian Academy of Sciences (Austria) 


\section{Session Chairs}

Security of Quantum Information Systems

Alexander Ling, Center for Quantum Technologies, National University of Singapore (Singapore)

Quantum Cryptography: Protocols and Implementations

Marco Lucamarini, Toshiba Research Europe Ltd. (United Kingdom) and Toshiba Corporation (Japan)

Technologies for Quantum Information Systems

Masato Koashi, The University of Tokyo (Japan)

Quantum Computation and Quantum Information Theory

Marcos Curty, Universidad de Vigo (Spain) 
Proc. of SPIE Vol. $9648964801-12$

Downloaded From: https://www.spiedigitallibrary.org/conference-proceedings-of-spie on 25 Apr 2023 Terms of Use: https://www.spiedigitallibrary.org/terms-of-use 